\title{
Proliferative Verrucous Leukoplakia - A Clinico Pathological Entity: An update
}

\author{
Keerthi Muddana ${ }^{1}$, Deepsagar Thadudari², \\ Aruna Kumari Maloth ${ }^{3}$, Gouri Pupala ${ }^{4}$, Nandan SRK ${ }^{5}$
}

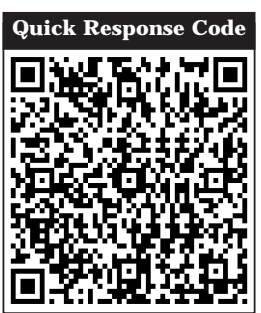

doi: $10.5866 / 2016.8 .10183$

${ }^{1}$ MDS, Reader

${ }^{2}$ Post graduate

${ }^{3 \& 4}$ Senior Lecturer

${ }^{5}$ Professor\& HOD

Department of Oral and Maxillofacial Pathology,

Kamineni Institute of Dental Sciences, Nalgonda,

Telangana, India.

\section{Article Info:}

Received: J uly 11, 2016

Review Completed: August 10, 2016

Accepted: September 9, 2016

Available Online: December, 2016 (www.nacd.in)

(c) NAD, 2016 - All rights reserved

\section{Email for correspondence:}

keerthikrishnamds@gmail.com.

\section{Introduction:}

White lesions are relatively frequent in oral cavity with a prevalence of approximately $24.8 \%$. Among them oral leukoplakia is quiet prevalent. Proliferative verrucous leukoplakia (PVL) is a rare form of oral leukoplakia, which was first described in 1985 by Hansen et al. According to the latest World Health Organization nomenclature, Oral proliferative verrucous leukoplakia conforms to the new terminology of "Potentially Malignant disorders" given that it is neither a deliminated lesion nor a condition. ${ }^{1}$ It is best defined as a

\section{ABSTRACT:}

Proliferative verrucous leukoplakia (PVL) is considered to be a progressive type of oral leukoplakia that is impossible to recognize in its nascent stages. The condition typically begins as a white plaque of hyper keratosis that eventually becomes a multifocal disease with confluent exophytic and proliferative features. It is observed most frequently in elderly women of fifth and six decades of life at the time of diagnosis. This article describes the clinical aspects and histological features and dealing with controversies in diagnosis.

Key words: leukoplakia, exophytic, proliferative

continuum of oral epithelial disease with hyperkeratosis at one end of a clinical and microscopic spectrum and verrucous carcinoma or squamous cell carcinoma at other end.

As reported in previous case series regarding PVL, women are affected more often than men. There is probably no racial preference. ${ }^{2}$ The mean age at the time of diagnosis is over 60 years. Tobacco use does not seem to have a significant influence on the disease given that PVL occurs both in smokers and non-smokers. ${ }^{3}$ The most common locations are the gingiva or alveolar ridge (often extending into 
the vestibule), tongue, and buccal mucosa. PVL often begins as a focal lesion spreading laterally over time and can be multifocal. Early in its course it is a flat hyperkeratotic lesion that becomes progressively verrucous and histologically often exhibits varying degrees of epithelial dysplasia. ${ }^{4}$

\section{Case Report:}

A 59-year-old female patient reported to the Department of Oral Medicine and Radiology with the chief complaint of rough white patch on right alveolar mucosa since 18 months. Shegavea history of chutta smoking since 40yrs. Intra oral examination revealed white verrucous slightly raised lesion with granular texture measuring $4 \mathrm{x}$ $2 \mathrm{cms}$ on right alveolar mucosa (Figure 1). The lesion was extending anterio posteriorly from mesial aspect of 47 to anterior mandibular right alveolar ridge. Bucco-lingually involves entire mandibular right alveolar ridge and extends to vestibule upto mesial aspect of 47. On pal pation the growth was firm, non tender, non fluctuant and non compressible. Extra oral examination revealed right submandibular Iymphadenopathy which was non tender and mobile. Based on the history and clinical examination a Provisional diagnosis of Proliferative verrucous leukoplakia was made with a differential diagnosis of Candidal leukoplakia, and verrucous carcinoma. The patient was subjected to foll owing investigations to reach to a final diagnosis Orthopantomograph (OPG), Complete hemogram, Incisional biopsy. Orthopantomograph reveals severe bone loss in relation to 47 and also showing that all remaining right mandibular teeth were missing. In complete hemogram all the values were in the normal range. An incisional biopsy was performed from right alveolar mucosa and the tissue was sent for histopathological analysis of mandibular multiple missing teeth and an incisional biopsy of the lesion was done for confirmation.

Histopathology showed marked hyperkeratosis with pseudoepitheliomatous hyperplasia with focal connective tissue entrapment. The surface of epithelium shows exophytic type of growth pattern with Parakeratin plugging. There is evidence of keratin pearl formation in few areas of the epithelium. Sub epithelial connective tissue shows mild lymphocytic infiltrate with no evidence of malignancy and was diagnosed as verrucous hyperplasia (Figure 2). Correlating clinical and histological features final diagnosis of Proliferative verrucous leukoplakia was given. The entire lesion was surgically excised. Later the follow-up was not possible as patient did not report back.

\section{Discussion:}

According to the latest WHO nomenclature, proliferative verrucous leukoplakia conforms to the new terminology of "potentially malignant disorders" given that it is neither a Deliminated lesion nor a condition. ${ }^{1}$ PVL presents with specific characteristics, mainly a more aggressive biological behavior than other forms of leukoplakia expressed by: A tendency toward multifocality; a high probability of recurrence; and a high rate of malignant transformation, which can range between $40 \%$ and $100 \%$ in a follow up period of 4.4 to 11.6 years. ${ }^{5}$ The lesions are slow growing yet persistent, as well as irreversible and resistant to all forms of treatment with a high recurrence rate. Throughout its development, it is common to find erythematous and/or verrucous areas that occasionally progress to verrucous carcinoma or squamous cell carcinoma. PVL is more common in elderly women who have had lesions of leukoplakia for many years. The mean age at the time of diagnosis is over 60 years. ${ }^{6}$ In the present case the patient was 59 yrs female and the growth was verrucous.

\section{Etiopathogenesis:}

PVL is of uncertain etiology. An association with human papilloma virus(HPV) infection, particularly 16 and 18 has been suggested. ${ }^{7}$ Pal efsky et al. (1995) studied nine lesions from seven patients with PVL and they found that eight lesions(89\%) were positive for HPV and out of these eight, seven contained HPV 16. They ther efore suggested that HPV 16 infection may play an important role in occurrence of these lesions. ${ }^{8}$ Aberrations in the cell cycle regulatory genes p16INK4a and p14ARF with homozygous del etion, loss of heterozygosity, and mutation appear to be frequent findings in PVL. ${ }^{8}$

\section{Histopathological features:}

The histol ogic findings are variable and usually a function of time or age of the lesion. The early lesions are deceptively bland exhibiting only hyperkeratosis, but over time they become progressively verrucous and often show varying degrees of epithelial dysplasia. Theadvanced lesions have a propensity for conventional squamous cell carcinoma and verrucous carcinoma within the histologic spectrum. ${ }^{9}$ 


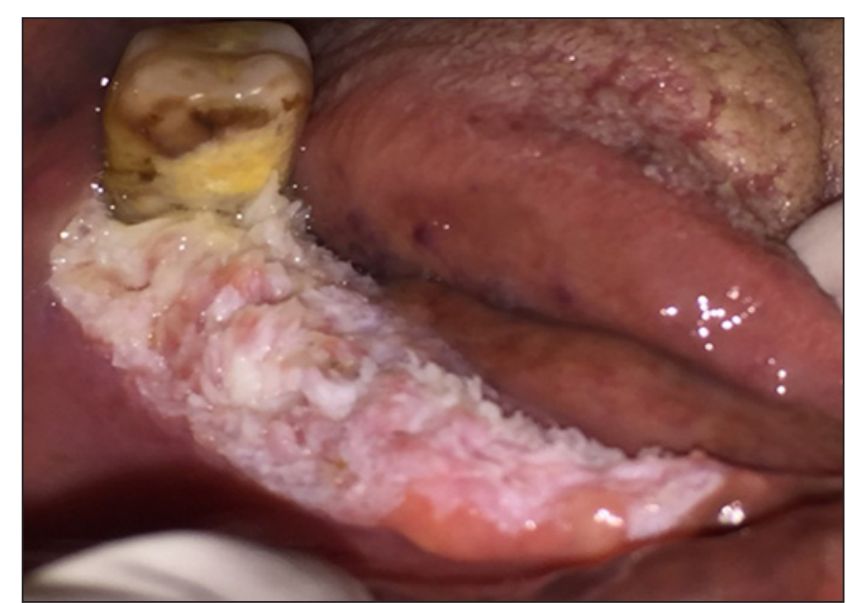

Figure 1: Verrucous growth on right alveolar mucosa

Hansen et al suggested histological stages in the continuum of PVL with intermediates as follows: ${ }^{10}$

Grade 0: Normal mucosa; Grade 2: Hyperkeratosis (clinical leukoplakia); Grade 4: Verrucous hyperplasia; Grade 6: Verrucous carcinoma; Grade 8: Papillary squamous cell carcinoma; Grade10: Less well differentiated squamous cell carcinoma.

Batsakis et al reduced the number of histological stages to four with intermediates. ${ }^{11}$ Grade 0: Clinical flat leukoplakia without dysplasia; Grade 2: Verrucous hyperplasia; Grade 4: Verrucous carcinoma; Grade 6: Conventional squamous cell carcinoma with intermediates.

\section{Diagnosis:}

Because of the lack of specific histological criteria, the diagnosis of PVL is based on combined clinical and histopathologic evidence of progression. In previously published series, diagnosis of PVL was made according to Hansen's definition. There are few studies that apply a set of diagnostic criteria that are mentioned as follows:

Ghazali et al., established the following criteria: ${ }^{6}$

- The lesion starts as homogenous leukoplakia without evidence of dysplasia at the first visit

- With time, some areas of leukoplakia become verrucous

- The disease progresses to the development of multiple isolated or confluent lesions at the same or a different site with time

- The disease progresses through the different

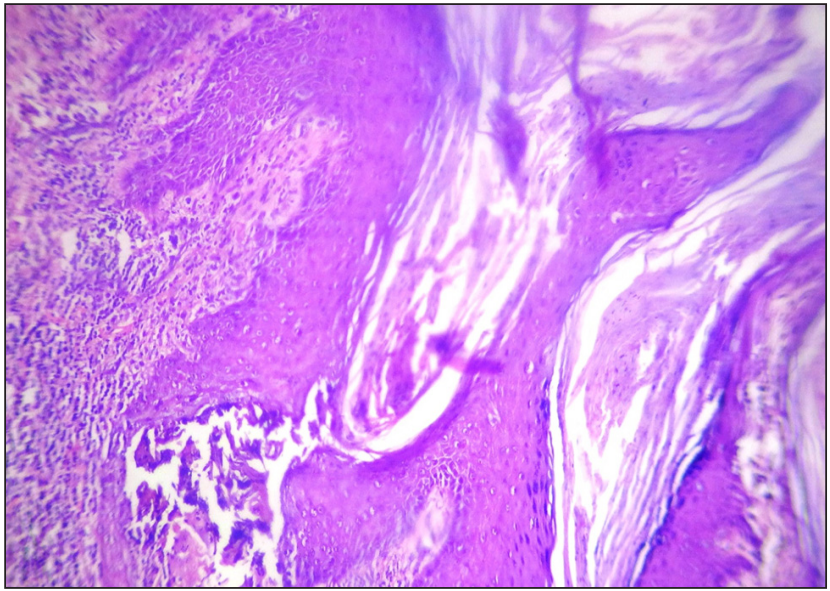

Figure 2: Hyperplastic epithelium showing parakeratin plugging along with sub epithelial inflammatory cell infiltrate (10x).

histopathological stages reported by Hansen et al

- The appearance of new lesions after treatment

- A follow up period of no less than one year Gandol fo et al established the following criteria for diagnosis: ${ }^{5}$

- An initially innocuous lesion characterized by a homogenous plaque that progresses over time to an exophytic, diffuse, usually multifocal, Iesion with a verrucous epithelial growth pattern

- Histopathologically, PVL changes gradually from a simple plaque of hyperkeratosis without dysplasia to verrucous hyperplasia, verrucous carcinoma or OSCC.

Cerero Lapiedra et al established the following major and minor criteria: ${ }^{12}$

\section{Major criteria:}

(a) Leukoplakia lesion with morethan two different oral sites, which is most frequently found in the gingiva, alveolar processes and palate

(b) The existence of a verrucous area

(c) That the lesions have spread or engrossed during development of the disease

(d) That there has been a recurrence in a previously treated area

(e) Histopathologically, there can be from simple epithelial hyperkeratosis to verrucous hyperplasia, Verrucous carcinoma or OSCC, whether in situ or infiltrating. 


\section{Minor criteria:}

(a) An OL lesion that occupies at least $3 \mathrm{~cm}$ when adding all the affected areas

(b) That the patient be female

(c) That patient (male or female) be a non smoker

(d) A disease evolution higher than 5 years.

In order to make the diagnosis of PVL, it was suggested that one of the two foll lowing combinations of the criteria mentioned before were met. Three major criteria ( $E$ being among them) or Two major criteria (E being among them) +two minor criteria. Nevertheless, at present, there is no criterion that will allow for the early diagnosis of the disease.

In present case it was fulfilling 4 major criteria and 2 minor criteria.

\section{Treatment:}

The lesions of PVL are persistent, progressive, and relentless and have a high recurrence rate regardless of the treatment method employed. Complete surgical excision is difficult because of the diffuse, multifocal nature of the condition and the inability to assess accurately surgical margins clinically and microscopically. Other modalities, such as laser ablation, external beam radiation, and chemotherapy, have been unsatisfactory. Methisoprinol as a synthetic agent capable of inhibiting viral ribonucleic acid synthesis and replication and of stimulating antiviral cell mediated reactions that have been shown to have some clinical efficacy in HPV - induced lesions. Topical photodynamic therapy also may prove useful. It causes relatively low morbidity ad no scarring and multiple mucosal sites can be treated simultaneously. ${ }^{13}$

\section{Malignant transformation and recurrences:}

PVL is characterized not only by a high rate of recurrences after treatment but also by malignant transformation in nearly $74 \%$ of cases, with a tendency for several oral cancers to appear. The gingiva and palate represented the areas with the highest frequency of these multiple malignant tumor. ${ }^{14}$

\section{Conclusion:}

The biologic behavior of PVL is decidedly more aggressive than the other forms of leukoplakia. However, the initial clinical presentation of PVL is so innocuous that one can easily dismiss it as insignificant until there is widespread oral involvement and carcinomatous transformation. Therefore, it is recommended to have the earliest possible diagnosis, as well as consensus on diagnostic criteria to achieve uniformity. To this effect, the present article mentioned a set of diagnostic criteria, which we believe should be applied to case series in order to verify its efficacy.

\section{References:}

1. Warnakulasuriya S, J ohnson NW, Van der Waal I. Nomenclature and classification of potentially malignant disorders of the oral mucosa. J Oral Pathol Med 2007; 36:575-80.

2. Cabay RJ, Morton TH J r, Epstein J B. Proliferative verrucous leukoplakia and its progression to oral carcinoma: a review of the literature. J Oral Pathol Med 2007; 36:25561.

3. Van der Waal I, Reichart PA. Oral proliferative verrucous leukoplakia revisited. Oral Oncol 2008; 44:719-21.

4. Thomas $\mathrm{P}$ Shopper, Robert B Brannon and William $\mathrm{H}$ Stalker. Proliferative verrucous leukoplakia: An aggressive form of oral leukoplakia. J Dent Hygiene 2004;78(3):1-6.

5. Gandolfo S, Castellani R, Pentenero M. Proliferative verrucous leukoplakia: A potentially malignant disorder olving periodontal sites. J Periodontol 2009; 80:27481.

6. Ghazali N, Bakri MM, Zain RB. Aggressive, multifocal oral verrucous leukoplakia: Proliferative verrucous leukoplakia or not? J Oral Pathol Med 2003; 32:38392.

7. Eversole LR. Papillary lesions of the oral cavity: Relationship to human papillomaviruses. J Calif Dent Assoc 2000; 28:9227.

8. Palefsky J M, Silverman S J r, Abdel Salaam M, Daniels TE, Greenspan J S. Association between proliferative verrucous leukoplakia and infection with human papillomavirus type 16. J Oral Pathol Med 1995; 24:1937.

9. Fettig A, Pogrel MA, Silverman S J r, Bramanti TE, Da Costa $M$, Regezi J A. Proliferative verrucous leukoplakia of the gingiva. Oral Surg Oral Med Oral Pathol Oral Radiol Endod 2000; 90:723-30.

10. Hansen LS, OIson J A, Silverman S J r. Proliferative verrucous leukoplakia. A long term study of thirty patients. Oral Surg Oral Med Oral Pathol 1985; 60:285•98.

11. Batsakis J G, Suarez P, el Naggar AK. Proliferative verrucous leukoplakia and its related lesions. Oral Oncol 1999; 35:354•9.

12. Cerero Lapiedra R, Balade Martinez D, Moreno Lopez LA, Esparza Gomez G, Bagan J V. Proliferative verrucous leukoplakia: A proposal for diagnostic criteria. Med Oral Patol Oral Cir Bucal 2010; 15:839• 45.

13. Klanrit $P$, Sperandio $M$, Brown $A L$, Shirlaw $P J$, Challacombe SJ, Morgan PR, et al. DNA ploidy in proliferative verrucous leukoplakia. Oral Oncol 2007; $43: 310 \cdot 6$.

14. Rakhi Issrani,Namdeo Prabhu, Vaishali keluskar .Oral Proloiferative Verrucous Leukoplakia : A Case Report with an update.Contemporary Clinical Dentistry 2013; 4(2):25862. 